\title{
Alpha - omega and omega - alpha phase transformations in zirconium under hydrostatic pressure : A 3D mesoscale study
}

\author{
Hemantha Kumar Yeddu ${ }^{\mathrm{a}, *}$, Hongxiang Zong ${ }^{\mathrm{a}, \mathrm{b}}$, Turab Lookman ${ }^{\mathrm{a}}$ \\ ${ }^{a}$ Theoretical Division, Los Alamos National Laboratory, Los Alamos, NM 87545, USA. \\ ${ }^{b}$ State Key Laboratory for Mechanical Behavior of Materials, Xian Jiaotong University, \\ Xian 710049, Peoples Republic of China.
}

\begin{abstract}
A three dimensional (3D) elastoplastic phase-field model is developed for modeling the hydrostatic pressure-induced alpha - omega phase transformation and the reverse phase transformation, i.e. omega - alpha, in zirconium (Zr). Plastic deformation and strain hardening of the material are also considered in the model. The microstructure evolution during both phase transformations is studied. The transformation start pressures at different temperatures are predicted and are plotted as a phase diagram. The effect of phase transformations on the mechanical properties of the material is also studied. The input data corresponding to pure $\mathrm{Zr}$ are acquired from experimental studies as well as by using the CALPHAD method. Our simulations show that three different omega variants form as laths. On release of pressure, reverse phase transformation initiates at lath boundaries. We observe that both phase transformations are martensitic in nature and also occur at the same pressure, i.e. little hysteresis. The transformation start pressures and the kinetics of the transformation predicted by our model are in good agreement with experimental results.
\end{abstract}

Keywords: Phase-field method, martensitic transformation, omega phase, microstructure, zirconium

\section{Introduction}

Zirconium $(\mathrm{Zr})$ and its alloys are used as structural materials in nuclear reactors and are also used in the chemical industry. These structural components need to withstand a wide range of temperatures and pressures. Thus the components have to be carefully fabricated by a combination of different thermomechanical processes such that a desired microstructure and mechanical properties are obtained. Different phase transformations and consequently different phases can occur in the component during its fabrication and during

*Corresponding Author. E-mail: hemy@lanl.gov, hemu23@gmail.com 
its service. This can adversely affect the mechanical properties and can lead to failure of the product. Hence, a thorough understanding of microstructural evolution and phase stability of Zr-alloys is essential.

The three phases that mainly form in $\mathrm{Zr}$-alloys are $\alpha, \beta$ and $\omega$ with crystal structures hexagonal close packed (hcp), body centered cubic (bcc) and hexagonal, respectively. In pure $\mathrm{Zr}$, the alpha phase $(\alpha)$ transforms to omega phase $(\omega)$ under high static pressure or during shock loading $[1,2]$, whereas at high temperatures the $\alpha$ phase transforms to $\beta$ phase [3]. The $\omega$ phase can also form from beta $(\beta)$ phase either athermally (by rapid quenching or stress-induced) or isothermally (by holding the material at a constant temperature below $773 \mathrm{~K}$ ) in $\mathrm{Zr}$-alloys [2, 4].

Zirconium, when used as a structural material in a nuclear reactor, can be subjected to high static pressure. This can lead to the formation of brittle $\omega$ phase and can affect the mechanical properties of the material. Hence, it is essential to study the co-evolution of microstructure and mechanical properties as well as measure the transformation start pressures during the hydrostatic pressure-induced $\alpha-\omega$ phase transformation in pure Zr using in-situ techniques. However, the difficulties associated with experimental set-ups in achieving pure hydrostatic conditions can affect the phase transformation kinetics as well as the transformation start pressures, due to the presence of shear stresses in the quasi-hydrostatic environment $[5,6]$.

Although constitutive models have been used to study the relation between phase transformation and plasticity [7-9], the recent advances in modeling the microstructure evolution by using the phase-field method $[10,11]$ are promising and can aid in the study of the $\alpha-\omega$ phase transformation under hydrostatic pressure. Several studies on solid state phase transformations using the phasefield approach have advanced this research area [12-32]. The development of the Ginzburg-Landau model coupled with Microelasticity theory, i.e. Phasefield microelasticity theory proposed by Khachaturyan [12], is an important advancement in this field. An alternative approach is developed by using the Ginzburg-Landau formulation in terms of strain order parameters and by enforcing the compatibility condition of strains to ensure that the dispalcement field is continuous $[13,20]$. This is in contrast to solving the displacement equations in terms of non-strain order parameters, as proposed by Khachaturyan [12]. Another important advancement is the inclusion of plasticity within the phase-field approach $[14,21,24,25,33]$. Due to these advances, it is possible to study the interplay between the martensitic microstructure evolution, internal stresses, strains and plastic strains. In our recent work, we have studied the athermal $\beta-\omega$ phase transformation in Zr-Nb alloys [26]. However, the $\alpha-\omega$ phase transformation in pure Zr under hydrostatic pressure has not been studied by using the phase-field approach.

In the present work the diffusionless hydrostatic pressure-induced alpha (hcp) to omega (hexagonal) phase transformation occurring in a single crystal of pure $\mathrm{Zr}$ is studied. A three dimensional (3D) phase-field model is developed by including plasticity and strain hardening within the Phase-field microelasticity theory $[14,21,25]$. The finite element method (FEM) is applied to solve the 
phase-field as well as the continuum mechanics equations. The effects of application and release of hydrostatic pressure on the microstructure evolution, as well as on the mechanical properties of the material, are studied. The transformation start pressures at different temperatures within the alpha-omega phase field are predicted. The phase diagram predicted by our model is consistent with that obtained from measurements using probes such as third generation light sources [34]. Our results also indicate that at $300 \mathrm{~K}$, both forward and reverse phase transformations occur in a martensitic manner and at the same pressure. On pressure release, reverse phase transformation initiates at lath boundaries. Negligible plastic strain is retained in the material after the reversion of $\omega$ to $\alpha$, which is in agreement with experimental results.

\section{Elastoplastic phase-field model}

Martensitic transformation can be modeled using the Cahn-Allen equation [35], also known as the Time-Dependent Ginzburg-Landau kinetic equation [12]. The microstructure evolution can be simulated by predicting the time-dependent variation of the phase-field variable $\eta_{p}$, which in turn is related to the minimization of the Gibbs energy $G$ of the system with respect to the phase-field variable as:

$$
\frac{\partial \eta_{p}}{\partial t}=-\sum_{q=1}^{q=v} L_{p q} \frac{\delta G}{\delta \eta_{q}}
$$

where $\frac{\delta G}{\delta \eta_{q}}$ is a variational derivative that serves as a driving force for the formation of the product phase denoted by the phase-field variable $\eta_{q}$, which is dependent on the position vector $\mathbf{r}$. $v$ is the total number of crystallographic orientations of the product phase and $L_{p q}$ is a kinetic parameter.

The phase transformation of alpha $(\alpha)$ (hexagonal close packed, hcp) to omega $(\omega)$ (hexagonal structure) causes asymmetry in the crystal structure and thereby gives rise to three possible crystallographic orientations (variants) of $\omega$. Thus, in order to represent the three possible variants, three phase-field variables, i.e. $\eta_{1}, \eta_{2}, \eta_{3}$, need to be considered in the model. Hence three phasefield equations, i.e. for $p=1,2,3$ and $v=3$ in Eq. (1), need to be solved at each time step. Each of the above three variants is governed by a stress-free transformation strain tensor $\epsilon_{i j}^{0}(p)$.

From a thermodynamic point of view, the Gibbs energy of a system undergoing a pressure-induced phase transformation consists of the following parts:

$$
G=\int_{V}\left(G_{v}^{c h e m}+G_{v}^{g r a d}+G_{v}^{e l}+G_{v}^{a p p l}\right) d V
$$

$G_{v}^{c h e m}$ corresponds to the chemical part of the Gibbs energy density of an unstressed system at the temperature under consideration. $G_{v}^{\text {grad }}$ is the extra Gibbs energy density caused by the interfaces. $G_{v}^{e l}$ is the elastic strain energy density, generated due to the structural phase transformation of $\alpha-\omega$. $G_{v}^{a p p l}$ is the extra Gibbs energy density caused by the externally applied load. 


\subsection{Chemical energy, $G_{v}^{\text {chem }}$}

The chemical part of the Gibbs energy density $G_{v}^{\text {chem }}$, expressed as a Landautype polynomial $[14,17]$, is given by:

$$
\begin{gathered}
G_{v}^{\text {chem }}\left(\eta_{1}, \eta_{2}, \eta_{3}\right)=\frac{1}{V_{m}}\left[\frac{1}{2} A\left(\eta_{1}^{2}+\eta_{2}^{2}+\eta_{3}^{2}\right)\right. \\
\left.-\frac{1}{3} B\left(\eta_{1}^{3}+\eta_{2}^{3}+\eta_{3}^{3}\right)+\frac{1}{4} C\left(\eta_{1}^{2}+\eta_{2}^{2}+\eta_{3}^{2}\right)^{2}\right]
\end{gathered}
$$

By considering the driving force $\Delta G_{m}$, i.e. the difference in the Gibbs energies of alpha and omega phases, and the Gibbs energy barrier $\Delta G^{*}$ terms in the above equation, the coefficients are modified [14] as: $A=32 \Delta G^{*}$, $B=3 A-12 \Delta G_{m}$ and $C=2 A-12 \Delta G_{m}$. Here $\Delta G^{*}=\frac{V_{m} \beta}{2 \delta^{2}}$, where $V_{m}$ is molar volume, $\delta$ is thickness of the interface and $\beta$ relates to interfacial energy and is discussed in the following section.

\subsection{Gradient Energy, $G_{v}^{\text {grad }}$}

The gradient energy density term, $G_{v}^{\text {grad }}$ can be expressed as [14, 17]:

$$
G_{v}^{\text {grad }}=\frac{1}{2} \sum_{p=1}^{p=v} \beta_{i j}(p) \frac{\partial \eta_{p}}{\partial r_{i}} \frac{\partial \eta_{p}}{\partial r_{j}}
$$

where $\mathbf{r}(\mathbf{x}, \mathbf{y}, \mathbf{z})$ is the position vector expressed in cartesian coordinates, $\beta_{i j}$ is the gradient coefficient matrix expressed in terms of the interfacial energy $\gamma$, molar volume $V_{m}$ and Gibbs energy barrier $\Delta G^{*}$. In the present work isotropic interfacial properties are considered, i.e. $\beta_{i j}$ is considered as a diagonal tensor with all the elements equal to $\beta$ [14], given as:

$$
\beta=\frac{9 \gamma^{2} V_{m}}{16 \Delta G^{*}}
$$

\subsection{Elastic energy, $G_{v}^{e l}$}

In order to calculate the elastic strain energy density $G_{v}^{e l}$, the elastic stress needs to be calculated by using the Microelasticity theory [12], which is briefly explained below. The martensitic transformation gives rise to deformation of the crystalline lattice and thereby induces stress-free transformation strains $\epsilon_{i j}^{0}(\mathbf{r})$ into the material. The surrounding parent matrix exerts strain $\epsilon_{i j}(\mathbf{r})$ on the product phase to resist the stress-free transformation strains and thereby induces elastic strain $\epsilon_{i j}^{e l}(\mathbf{r})$, which in turn gives rise to elastic stress $\sigma_{i j}(\mathbf{r})$, in the material. When the elastic stress exceeds the yield limit of the material, plastic deformation initiates and gives rise to plastic strain $\epsilon_{i j}^{p l}(\mathbf{r})$. The actual strain tensor $\epsilon_{i j}(\mathbf{r})$ is linearly related to the local displacement vector $\mathbf{u}(\mathbf{r})$, which is obtained by solving the mechanical equilibrium condition.

The elastic strain energy density term $G_{v}^{e l}$ can be expressed as:

$$
G_{v}^{e l}=\int_{\epsilon_{i j}^{0}(\mathbf{r})}^{\epsilon_{i j}(\mathbf{r})} \sigma_{i j}(\mathbf{r}) d \epsilon_{i j}(\mathbf{r})
$$


where $\epsilon_{i j}^{0}(\mathbf{r})$ is the stress-free transformation strain, $\epsilon_{i j}(\mathbf{r})$ is the total strain and $\sigma_{i j}(\mathbf{r})$ is the elastic stress. We have considered isotropic elastic properties and hence $\sigma_{i j}(\mathbf{r})$ is given by:

$$
\sigma_{i j}(\mathbf{r})=\frac{E}{1+\nu}\left[\epsilon_{i j}^{e l}(\mathbf{r})+\frac{\nu}{1-2 \nu} \epsilon_{k k}^{e l}(\mathbf{r}) \delta_{i j}\right]
$$

where $E$ is Youngs modulus of elasticity and $\nu$ is Poisson ratio and $\epsilon_{k l}^{e l}(\mathbf{r})$ is the elastic strain, given by:

$$
\epsilon_{k l}^{e l}(\mathbf{r})=\epsilon_{k l}(\mathbf{r})-\epsilon_{k l}^{0}(\mathbf{r})-\epsilon_{k l}^{p l}(\mathbf{r})
$$

where $\epsilon_{k l}^{p l}(\mathbf{r})$ is the plastic strain.

The total strain $\epsilon_{i j}(\mathbf{r})$ is given by:

$$
\epsilon_{i j}(\mathbf{r})=\frac{1}{2}\left(\frac{\partial u_{i}(\mathbf{r})}{\partial r_{j}}+\frac{\partial u_{j}(\mathbf{r})}{\partial r_{i}}\right)
$$

where $\mathbf{u}(\mathbf{r})$ is the local displacement vector obtained by solving the mechanical equilibrium condition:

$$
\frac{\partial \sigma_{i j}(\mathbf{r})}{\partial r_{j}}=0
$$

The stress-free transformation strain (SFTS) is given by:

$$
\epsilon_{i j}^{0}(\mathbf{r})=\sum_{p=1}^{p=v} \eta_{p}(\mathbf{r}) \epsilon_{i j}^{0}(p)
$$

where $\epsilon_{i j}^{0}(p)$ is the SFTS tensor corresponding to each variant.

Based on the recent in-situ synchrotron X-ray diffraction measurements $[3,36]$, we have chosen Silcock transformation pathway for the $\alpha-\omega$ transformation. According to the Silcock mechanism and the corresponding crystallographic orientation relationships between $\alpha$ and $\omega$ variants (Fig. 1), SFTS tensors of the three $\omega$ variants are:

$$
\begin{aligned}
\epsilon_{i j}^{0}(1) & =\left[\begin{array}{ccc}
\epsilon_{1} & 0 & 0 \\
0 & \epsilon_{2} & 0 \\
0 & 0 & \epsilon_{3}
\end{array}\right], \epsilon_{i j}^{0}(2)=\left[\begin{array}{ccc}
\frac{\epsilon_{1}+3 \epsilon_{3}}{4} & 0 & \frac{\sqrt{3}\left(-\epsilon_{1}+\epsilon_{3}\right)}{4} \\
0 & \epsilon_{2} & 0 \\
\frac{\sqrt{3}\left(-\epsilon_{1}+\epsilon_{3}\right)}{4} & 0 & \frac{3 \epsilon_{1}+\epsilon_{3}}{4}
\end{array}\right], \\
\epsilon_{i j}^{0}(3) & =\left[\begin{array}{ccc}
\frac{\epsilon_{1}+3 \epsilon_{3}}{4} & 0 & \frac{\sqrt{3}\left(\epsilon_{1}-\epsilon_{3}\right)}{4} \\
0 & \epsilon_{2} & 0 \\
\frac{\sqrt{3}\left(\epsilon_{1}-\epsilon_{3}\right)}{4} & 0 & \frac{3 \epsilon_{1}+\epsilon_{3}}{4}
\end{array}\right]
\end{aligned}
$$

where $\epsilon_{1}=\frac{c_{\omega}-a_{\alpha}}{a_{\alpha}}, \epsilon_{2}=\frac{a_{\omega}-c_{\alpha}}{c_{\alpha}}, \epsilon_{3}=\frac{2 \sqrt{3} a_{\omega}-3 \sqrt{3} a_{\alpha}}{3 \sqrt{3} a_{\alpha}} . a_{\alpha}, c_{\alpha}, a_{\omega}$ and $c_{\omega}$ are lattice constants of $\alpha$ and $\omega$ phases, respectively. The SFTS tensors of variants- 2 and 
3 in Eq. (12) are obtained by using $\epsilon_{i j}^{0}(2)=A \epsilon_{i j}^{0}(1) A^{T}$ and $\epsilon_{i j}^{0}(3)=A \epsilon_{i j}^{0}(2) A^{T}$, where $\mathrm{A}$ is rotation matrix given by:

$$
A=\left[\begin{array}{ccc}
\frac{1}{2} & 0 & -\frac{\sqrt{3}}{2} \\
0 & 1 & 0 \\
\frac{\sqrt{3}}{2} & 0 & \frac{1}{2}
\end{array}\right]
$$

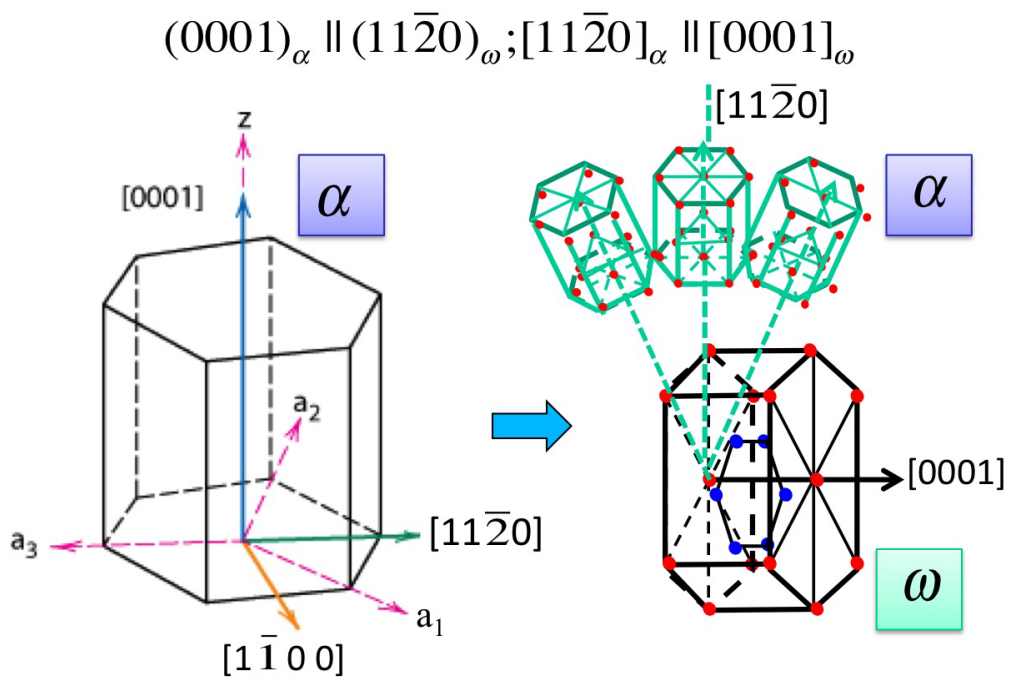

Figure 1: Orientation relationship between alpha and omega phases according to the Silcock mechanism, $(0001)_{\alpha} \|(11 \overline{2} 0)_{\omega}$ and $[11 \overline{2} 0]_{\alpha} \|[0001]_{\omega}$.

The plastic strain comes into existence only when the elastic stress exceeds the yield limit, thereby initiating plastic deformation that acts as a relaxation of the elastic stress. The von Mises criterion is employed to check whether the elastic stress has exceeded the yield stress $\left(\sigma_{y}\right)$. According to the von Mises criterion, plastic deformation occurs if:

$$
\begin{aligned}
\bar{\sigma}^{2}-\sigma_{y}^{2} & =\frac{1}{2}\left\{\left(\sigma_{x x}-\sigma_{y y}\right)^{2}+\left(\sigma_{y y}-\sigma_{z z}\right)^{2}\right. \\
& \left.+\left(\sigma_{z z}-\sigma_{x x}\right)^{2}\right\}+3\left(\sigma_{x y}^{2}+\sigma_{y z}^{2}+\sigma_{z x}^{2}\right)-\sigma_{y}^{2} \geq 0
\end{aligned}
$$

where $\bar{\sigma}$ is von Mises equivalent stress.

The evolution of the plastic strain field [14, 24, 33] can be calculated by:

$$
\frac{\partial \epsilon_{i j}^{p l}(\mathbf{r})}{\partial t}=-k_{i j k l} \frac{\delta G_{v}^{\text {shear }}}{\delta \epsilon_{k l}^{p l}(\mathbf{r})}
$$

where $\epsilon_{i j}^{p l}(\mathbf{r})$ is the local plastic strain, $G_{v}^{\text {shear }}$ is shear energy density and $k_{i j k l}$ $\left(=k c_{i j k l}^{-1}\right)$ is plastic kinetic coefficient. $c_{i j k l}^{-1}$ denotes the compliance tensor and 
$k$ is a parameter, which controls the rate at which the elastic stresses are relaxed by means of plastic deformation and is called plastic relaxation rate.

The shear energy density $G_{v}^{\text {shear }}$ is given by:

$$
G_{v}^{\text {shear }}=\int_{e_{i j}^{0}(\mathbf{r})}^{e_{i j}(\mathbf{r})} c_{i j k l}\left(e_{k l}(\mathbf{r})-e_{k l}^{0}(\mathbf{r})\right) d e_{i j}(\mathbf{r})
$$

where $c_{i j k l}$ is the tensor of elastic constants, $e_{i j}(\mathbf{r})$ and $e_{i j}^{0}(\mathbf{r})$ are the deviatoric total strain tensor and deviatoric stress-free transformation strain tensors given by:

$$
\begin{gathered}
e_{i j}(\mathbf{r})=\epsilon_{i j}(\mathbf{r})-\frac{1}{3} \epsilon_{k k}(\mathbf{r}) \delta_{i j} \\
e_{i j}^{0}(\mathbf{r})=\epsilon_{i j}^{0 t o t}(\mathbf{r})-\frac{1}{3} \epsilon_{k k}^{0 t o t}(\mathbf{r}) \delta_{i j}
\end{gathered}
$$

where $\epsilon_{i j}^{0 t o t}(\mathbf{r})$ is given by:

$$
\epsilon_{i j}^{0 t o t}(\mathbf{r})=\epsilon_{i j}^{0}(\mathbf{r})+\epsilon_{i j}^{p l}(\mathbf{r})
$$

Linear isotropic strain hardening is considered by using the following expression [37]:

$$
\sigma_{y}=\sigma_{y}^{0}+H \epsilon^{p l}(\mathbf{r})
$$

where $\sigma_{y}$ is the yield stress of the material that depends on the plastic strain, $\sigma_{y}^{0}$ is the initial yield stress, $H$ is the hardening modulus and $\epsilon^{p l}(\mathbf{r})$ is the von Mises equivalent plastic strain.

\subsection{Extra Gibbs energy due to application of external load, $G_{v}^{a p p l}$}

The extra Gibbs energy density $G_{v}^{a p p l}$ caused by the externally applied load can be expressed as [21]:

$$
G_{v}^{a p p l}=-\sigma_{i j}^{a p p l} \epsilon_{i j}^{0}(\mathbf{r})
$$

where $\sigma_{i j}^{a p p l}$ is the externally applied Cauchy stress tensor, given by:

$$
\sigma_{i j}^{a p p l}=\left[\begin{array}{lll}
\sigma_{x x} & \sigma_{x y} & \sigma_{x z} \\
\sigma_{y x} & \sigma_{y y} & \sigma_{y z} \\
\sigma_{z x} & \sigma_{z y} & \sigma_{z z}
\end{array}\right]
$$

\section{Simulations}

We study the effect of hydrostatic pressure on omega phase formation as well as on the microstructure evolution by considering a single crystal of pure Zr. We use a small embryo of omega phase pre-existing in a single crystal of alpha phase to initiate the phase transformation, in agreement with the existing literature [38]. In order to simulate the hydrostatic pressure loading, the diagonal terms in Eq. (21) are considered to be equal to each other $\left(\sigma_{x x}=\sigma_{y y}\right.$ $=\sigma_{z z}=-\mathrm{P}$, where $\mathrm{P}$ is the absolute value of the applied pressure) and all the 
off-diagonal terms are considered to be equal to zero. After the phase transformation as well as the material reach the equilibrium state, another simulation is performed by releasing the hydrostatic pressure in order to study the omega phase reversion kinetics as well as the microstructure evolution. We have also performed several simulations to predict the transformation start pressures at different temperatures.

Table 1 shows the input data for the simulations.

Table 1: Simulation data.

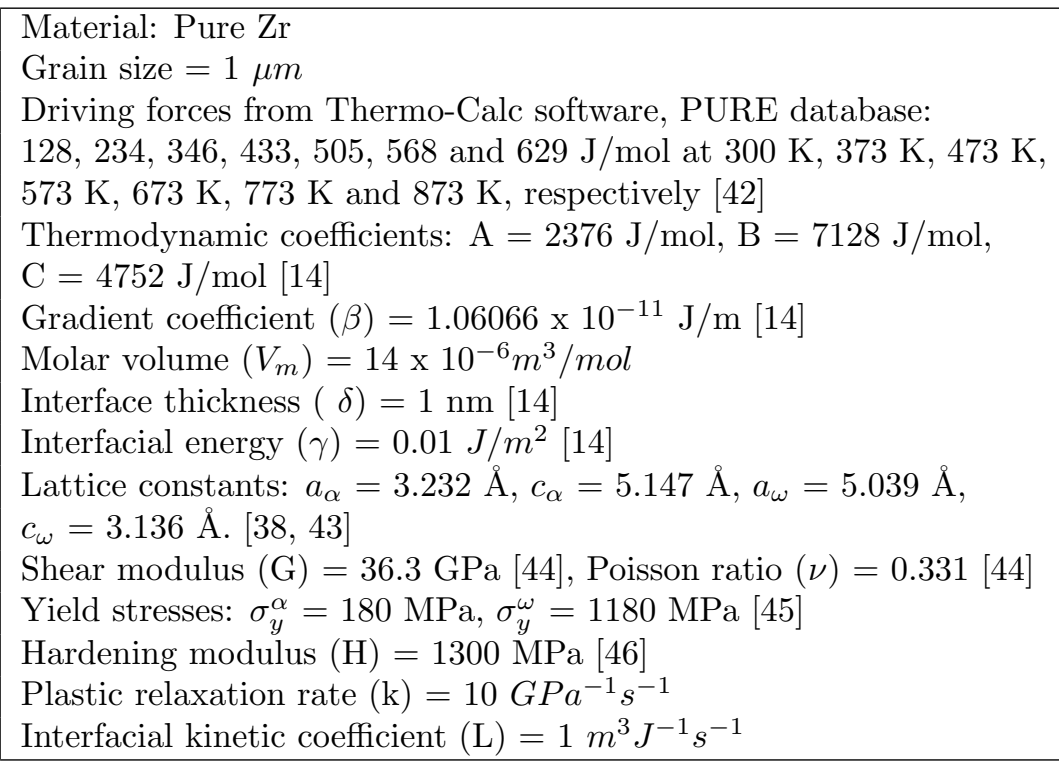

We have performed different simulations in order to study the sensitivity of the results with respect to input data, e.g. yield limits, interfacial energy. Our results show that when the yield limit of $\alpha$ is extremely high $\left(10^{6} \mathrm{MPa}\right)$, the material does not undergo plastic deformation. As a consequence, the internal stresses overcome the net available driving force for formation of $\omega$ and hence the phase transformation does not occur. In the case of a very low yield limit $\left(10^{-6} \mathrm{MPa}\right)$, plastic deformation occurs relatively easily compared to the other case and thereby provides the necessary stress relaxation for the formation of $\omega$.

The interfacial energy $(\gamma)$ affects the Gibbs energy barrier $\left(\Delta G^{*}\right)$ (Eq. (5)), which in turn affects the coefficients A, B and C (Sec. 2.1). With a very high interfacial energy $\left(100 \mathrm{~J} / \mathrm{m}^{2}\right)$, the Gibbs energy barrier becomes extremely large and hence the transformation never occurs. With a very low interfacial energy $\left(10^{-6} \mathrm{~J} / \mathrm{m}^{2}\right)$, the Gibbs energy barrier becomes too small such that the transformation can occur without any driving force. Hence we have selected an appropriate value for the interfacial energy, based on the interfacial energy values 
used to model solid-state phase transformations in other metallic materials $[12$, $14,17,39]$. Moreover, the interfacial energy is assumed to be same for $\alpha-\omega$ as well as $\omega-\alpha$ interfaces.

As the experimental data related to the mobility of the martensitic interface is ambiguous, the matrix of kinetic parameters $L_{p q}$ in Eq. (1) that governs the mobility of the martensitic interface is considered to be an identity matrix. By using the relation $t^{*}=\frac{t L R T}{V_{m}}$ and the input data given in Table-1, we can estimate the real time $(\mathrm{t})$ needed for the completion of transformation, e.g. approximately 2.8 microseconds (for $\mathrm{t}^{*}=500$ ) at $300 \mathrm{~K}$ and $4 \mathrm{GPa}$. However, due to the ambiguity associated with $L$, we express the time scales only in terms of dimensionless time $t^{*}$.

The entire mathematical formulation is solved using tetrahedral finite elements and by using Dirichlet boundary conditions. Computations are performed on a mesh with 50x50x50 grid points by using FemLego software [40, 41].

\section{Results and discussion}

\subsection{Microstructure evolution under hydrostatic pressure}

Our model predicts that $\omega$ forms in $\mathrm{Zr}$ at $300 \mathrm{~K}$ under a hydrostatic pressure of $4 \mathrm{GPa}$. The microstructure evolution is shown in Fig. 2. The iso-surfaces of the three phase-field variables $\left(\eta_{p}=0.5\right)$, i.e. $\omega$ variants- $1,2,3$, are shown in red, blue, and green, respectively. On application of hydrostatic pressure, the pre-existing spherical embryo of variant-1 grows in the shape of a plate due to the elastic energy contributions [14]. As the variant grows further, it transforms into a lath-like unit (Fig. 2). The growth of variant-1 leads to stresses in the material, which are minimized by the nucleation of variants- 2 and 3 . This process, known as autocatalysis [14], continues and one can see the nucleation and growth of many different $\omega$ variants. Plastic deformation of the matrix $\alpha$ occurs due to the growth of brittle $\omega$ and relaxes the stresses built in the material due to the phase transformation.

Different authors have observed different morphologies of $\omega$ during static pressure loading experiments. Banerjee et al. have observed that the entire $\alpha$ grain transforms into an $\omega$ grain when $\mathrm{Zr}$ is soaked for 24 hours [2]. Rabinkin et al. have observed ellipsoidal $\omega$ particles in $\mathrm{Zr}$ when soaked under pressure for 4 hours, a morphology that can be observed during the $\beta-\omega$ phase transformation in $\mathrm{Zr-Nb}$ alloys [47]. Zr under shock loading exhibits a plate-like morphology $[48]$.

\subsection{Microstructure evolution during pressure release}

On release of hydrostatic pressure, $\omega$ becomes unstable and $\alpha$ becomes the stable phase and hence $\omega$ reverts to $\alpha$ (Fig. 3). Several experimental studies have shown that only a fraction of $\omega$ reverts to $\alpha$ [49]. However, we observe that $\omega$ completely reverts to $\alpha$, with a negligible volume fraction $(0.01 \%)$ of $\omega$ retained after the reversion. As the simulated single crystal does not contain any defects and impurities, which can oppose the phase reversion as in the case 


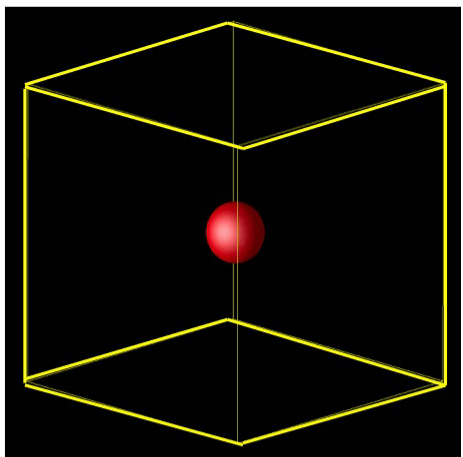

(a)

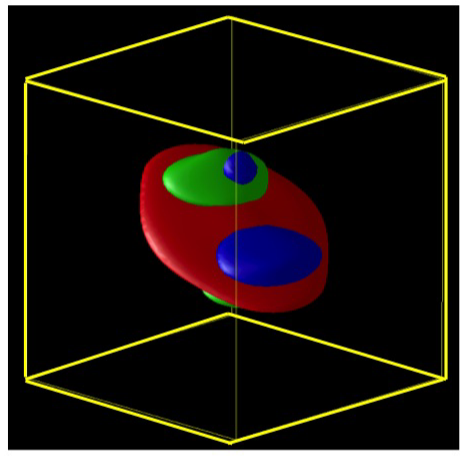

(c)

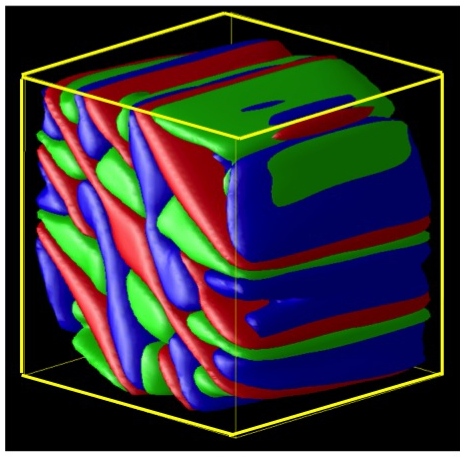

(e)

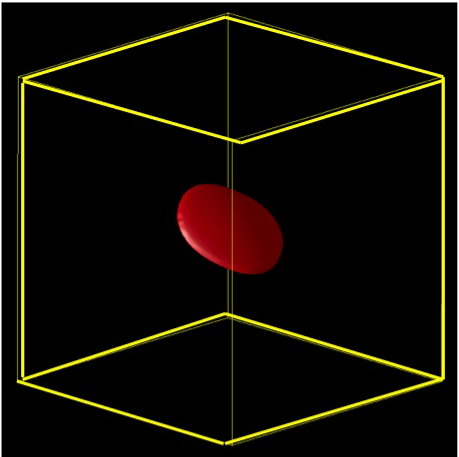

(b)

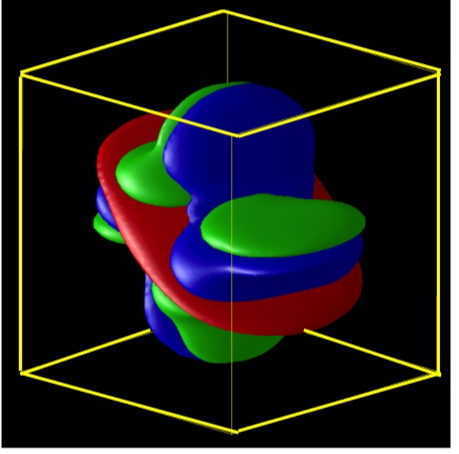

(d)

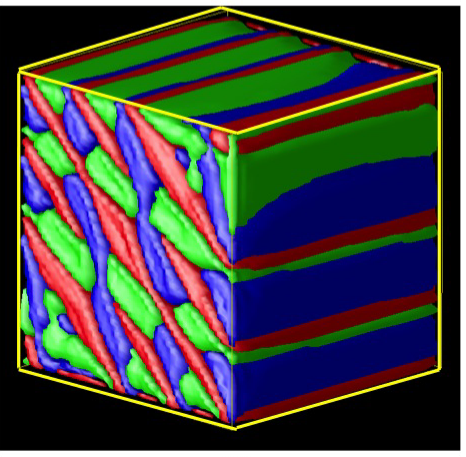

(f)

Figure 2: Microstructure evolution at (a) $t^{*}=0$ (b) $t^{*}=50$ (c) $t^{*}=60$ (d) $t^{*}=70$ (e) $t^{*}=100$ (f) $\mathrm{t}^{*}=500 \mathrm{in} \mathrm{Zr}$ under hydrostatic pressure of $4 \mathrm{GPa}$ at $300 \mathrm{~K}$. Iso-surfaces of phase-field variables $\eta_{1}, \eta_{2}, \eta_{3}$ corresponding to the three different $\omega$ variants are shown in red, blue, and green, respectively. $\alpha$ is shown in black inside the simulation domain. $\omega$ variants grow as lath-like units. Autocatalysis and plastic deformation facilitate the phase transformation.

of a real material, we observe a complete reversion. Errandonea et al. have observed that defects can oppose the complete reversion of $\beta$ to $\alpha$ and instead give rise to a mixture of $\alpha$ and $\omega$ in $\mathrm{Ti}[6]$. 


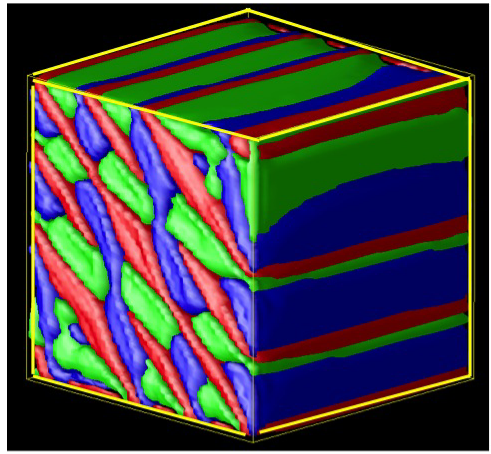

(a)

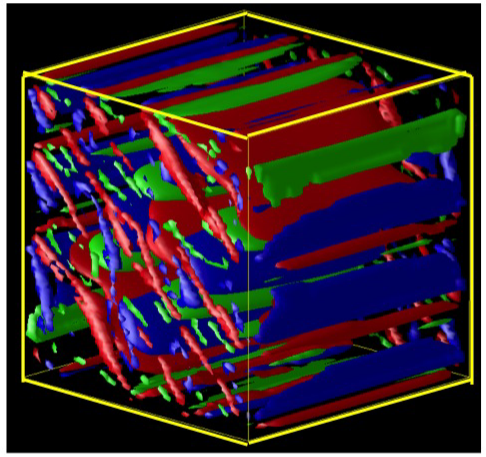

(c)

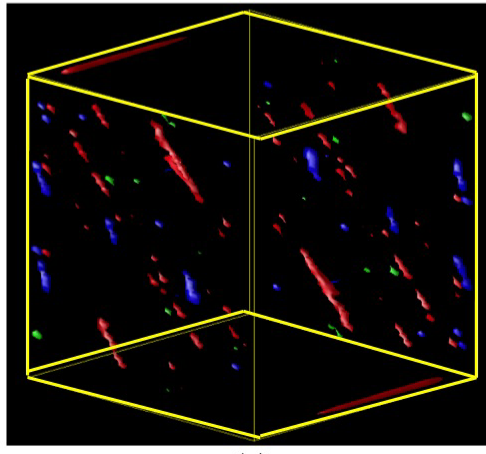

(e)

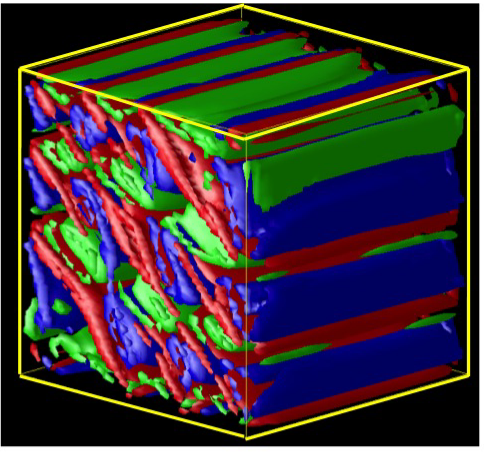

(b)

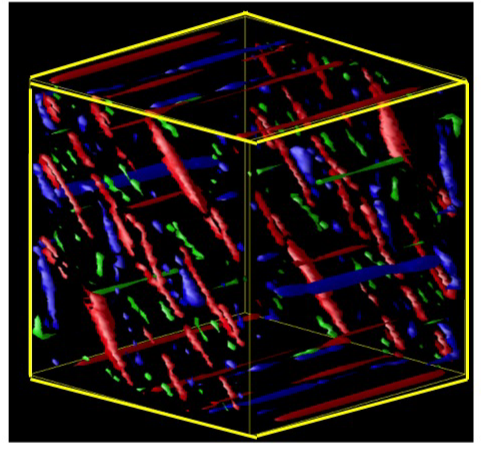

(d)

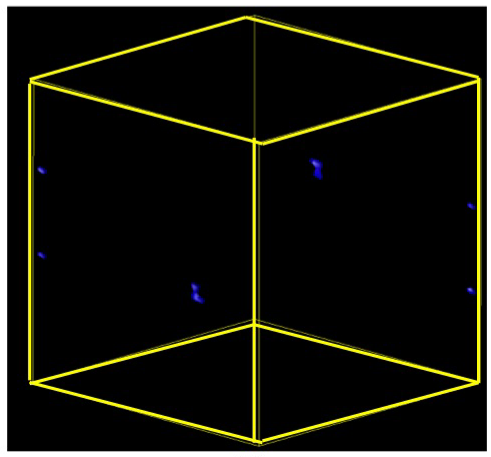

(f)

Figure 3: Microstructure evolution after release of the hydrostatic pressure, at (a) $\mathrm{t}^{*}=500(\mathrm{~b})$ $\mathrm{t}^{*}=505(\mathrm{c}) \mathrm{t}^{*}=510(\mathrm{~d}) \mathrm{t}^{*}=550(\mathrm{e}) \mathrm{t}^{*}=600(\mathrm{f}) \mathrm{t}^{*}=1000$. The $\omega$ phase completely reverts to $\alpha$.

We observe that the transformation start pressure for reversion $(\omega-\alpha)$ is $4 \mathrm{GPa}$, i.e. same as that for the forward phase transformation $(\alpha-\omega)$. The phase transformation is aided by shear forces due to its martensitic nature. The presence of shear forces reduces the hysteresis and hence the forward and reverse phase transformations occur at the same pressure [49]. Our results are 
in accordance with experimental results of Zilbershtein et al., although their transition pressure for both forward and backward transformations is $2.2 \pm$ $0.3 \mathrm{GPa}[49]$. The imperfect regions in polycrystalline experimental samples may lose stability at lower driving forces (transformation pressures) because of locked-in energy in them. Hence, the transformation pressures predicted by our single crystal model are slightly higher than the experimental values. This is in agreement with the comparisons of theoretical and experimental transformation pressures by Vohra [38].

The kinetics of reversion from a front view of the crystal and the corresponding von Mises equivalent plastic strain plots are shown in Fig. 4. Reversion initiates near the lath boundaries or at inter lath boundaries, as shown in Figs. 3 and 4, and progresses inwards. Since the lath boundaries are the areas where a low nucleation energy is sufficient to overcome the transformation barriers, reversion occurs primarily at the lath boundaries. These results are in good agreement with our study on reversion of martensite to austenite in stainless steels [28].

The lath boundaries and inter lath boundaries are the areas with relatively larger plastic strains compared to the cores of the laths (Fig. 4a and b). During the $\alpha-\omega$ phase transformation, the $\omega$ variants (laths) grow by deforming the $\alpha$ matrix and induce internal stresses, strains and plastic strains in the $\alpha$ matrix. Hence plastic strains are concentrated at the lath boundaries. As the reverse phase transformation $(\omega-\alpha)$ progresses, the volume fraction of $\alpha$ (shown in white in Fig. 4c, e, g) starts to increase whereas the volume fraction of $\omega$ decreases by inward motion of lath boundaries. Since $\alpha$ is a softer phase compared to $\omega$, growth of $\alpha$ relaxes the plastic strains. Hence plastic strains decrease in the newly formed $\alpha$ regions. Moreover, the omega variants are under compressive stress, exerted by the growing $\alpha$. Therefore, we observe that plastic strains are concentrated only inside the $\omega$ variants and not in the reversed $\alpha$ regions (Fig. 4c -f). Plastic strains decrease to negligible levels when most of the $\omega$ reverts to $\alpha$ (Fig. $4 \mathrm{~g}$ and $\mathrm{h}$ ).

In the case of steels, where martensitic transformation and reversion are associated with significant amount of plastic deformation of the material, we observed that a large amount of plastic strains are retained after the reverse phase transformation [28]. The amount of plastic deformation in $\mathrm{Zr}$ is not as large as that in steels. Hence we observe that most of the plastic strains are completely reversible and decrease to negligible levels after the reversion of $\omega$ to $\alpha$ (Fig. $4 \mathrm{~g}$ and $\mathrm{h}$ ).

Wenk et al. [3] have shown that the static pressure-induced $\omega$ in $\mathrm{Zr}$, when subjected to heat treatment, reverts to $\alpha$. Their results show that the reversed $\alpha$ retains the texture of the original $\alpha$, indicating perfect memory (orientation memory). During reversion, as the material is unable to retain plastic strains that were generated during the $\alpha-\omega$ phase transformation, it can retain its original texture. Our results show that negligible amount of plastic strains are retained in the material after reversion, which is in good agreement with Wenk et al.'s results. Although Wenk et al.'s experiments involved heat treatments to revert $\omega$, whereas our simulated reversion occurs by releasing the applied 

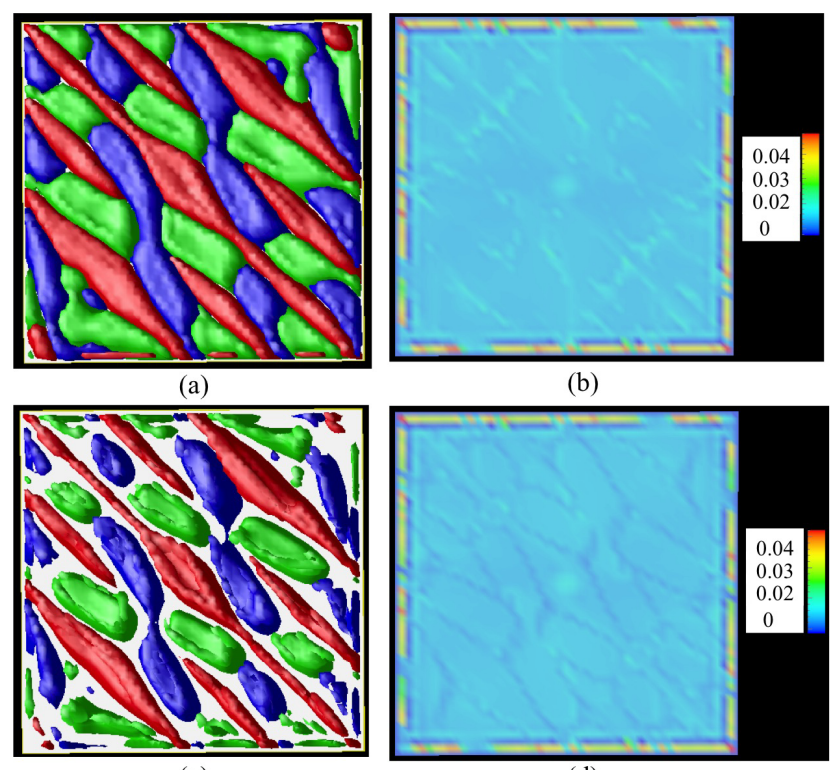

(b)
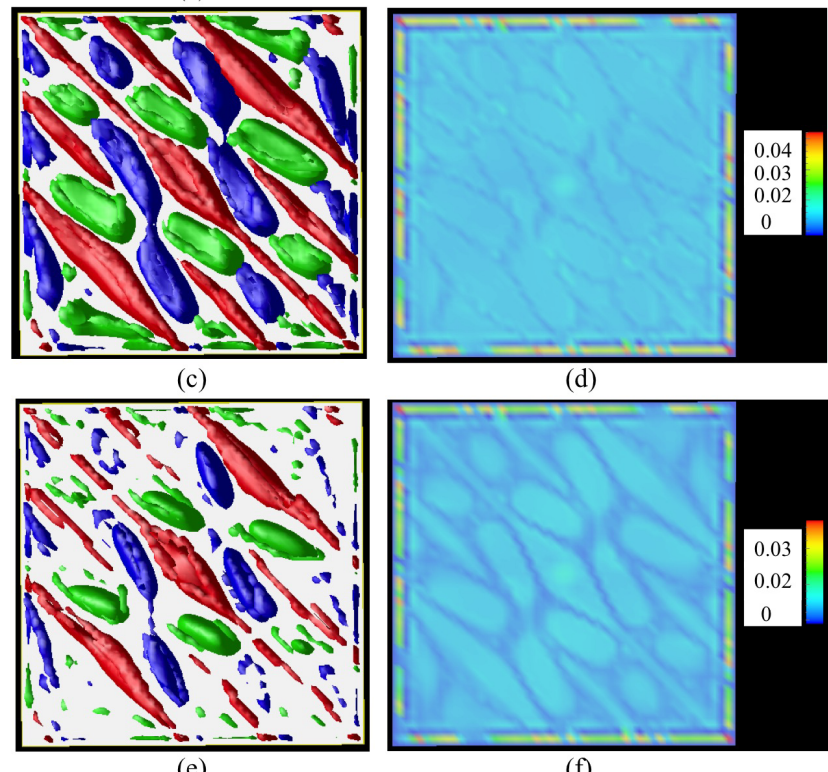

(d)
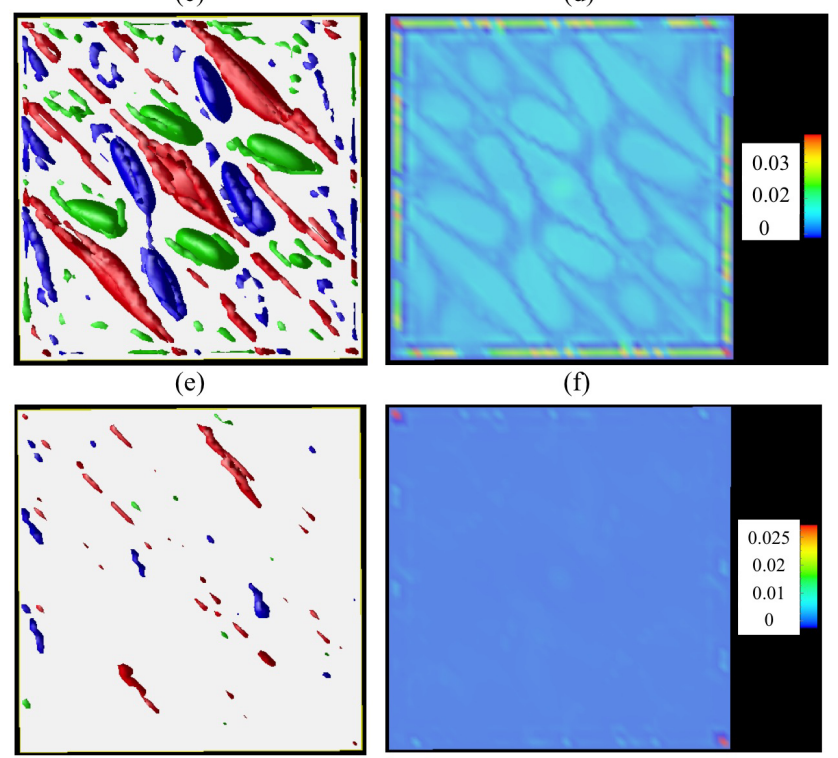

(g)

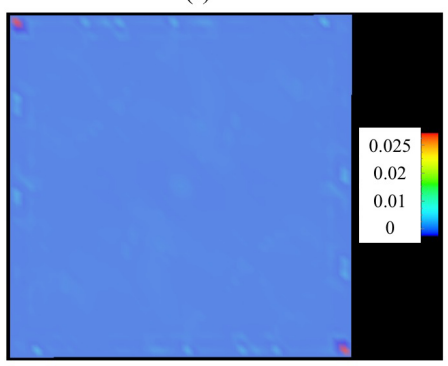

(h)

Figure 4: Evolution of microstructure and the corresponding von Mises equivalent plastic strain after release of the hydrostatic pressure, at (a), (b) $t^{*}=500$ (c), (d) $t^{*}=505$ (e), (f) $\mathrm{t}^{*}=510(\mathrm{~g}),(\mathrm{h}) \mathrm{t}^{*}=600 . \alpha$ is shown in white, whereas $\omega$ variants are shown in red, blue and green in (a), (c), (e), (g). Reversion initiates at inter lath boundaries and progresses inwards.

hydrostatic pressure, the overall material behavior is in good agreement. 


\subsection{Prediction of phase diagram}

For each given temperature, different simulations are performed at different pressures and the transformation start pressures are predicted. The simulation results along with experimental data of Zhang et al. [34] and Wenk et al. [3] are plotted as a phase diagram (Fig. 5). The equilibrium phase boundaries shown in the figure are obtained from Ref. [34].

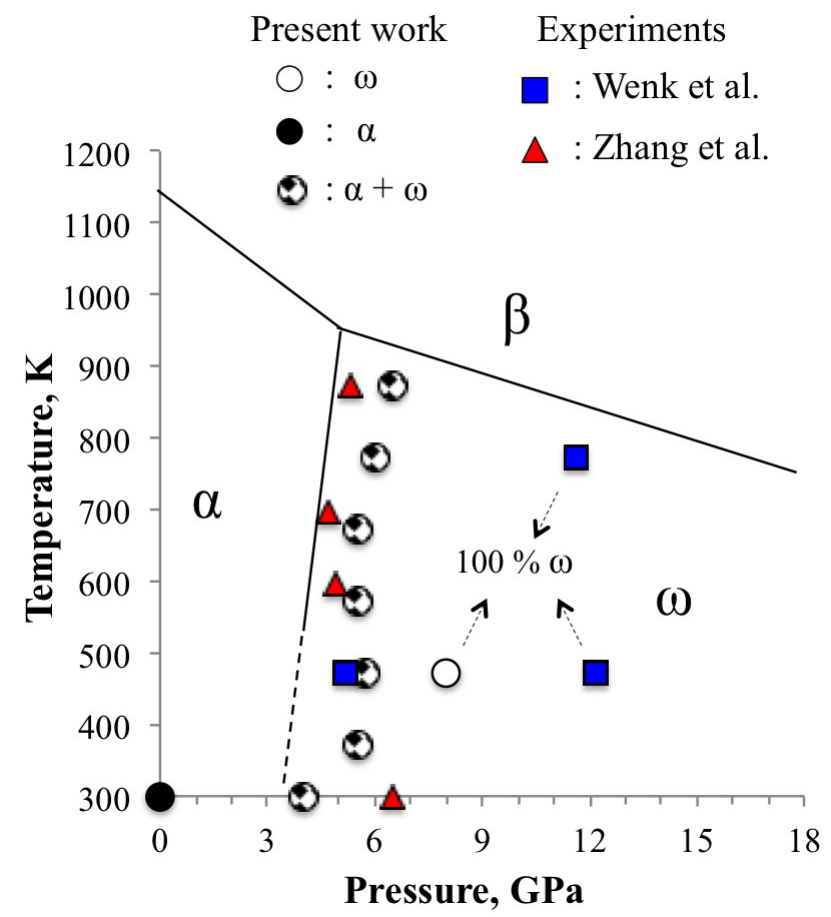

Figure 5: Phase diagram showing the equilibrium phase boundaries [34] and the transformation pressures estimated by the present model as well as the experimental studies of Wenk et al. [3] and Zhang et al. [34]. Transformation pressures predicted by the model are consistent with experimental results.

Our results show that at room temperature $(300 \mathrm{~K}) \omega$ is formed at a hydrostatic pressure of $4 \mathrm{GPa}$. Although $\alpha$ does not transform completely and the microstructure is a mixture of $\alpha$ and $\omega$, the dominant phase in the microstructure is $\omega$ with $90 \%$ volume fraction. Several experimental studies report that the $\alpha-\omega$ phase transformation occurs at various pressures in the range of 2.0 $-6.0 \mathrm{GPa}$ and the equilibrium transition at ambient temperature is believed to be at $2.2 \mathrm{GPa}[5,38]$. The extrapolated equilibrium phase boundaries in Fig. 5 show that the transition pressure at ambient temperature is $3.4 \mathrm{GPa}$ [34]. Zhang et al.'s experiments show that the $\alpha-\omega$ phase transformation can occur between 5.5 and $6.5 \mathrm{GPa}$ [34]. Wenk et al.'s diamond anvil cell (DAC) experiments did not show any $\omega$ phase formation at $3.8 \mathrm{GPa}$ and $298 \mathrm{~K}$ [3]. Liu 
et al. have reported a transition pressure of $5 \mathrm{GPa}$ at room temperature [50]. Olinger et al. [43] have measured the $\omega$-start pressure to be $3.9 \mathrm{GPa}$ at $297 \mathrm{~K}$. The transformation start pressure of $4 \mathrm{GPa}$ at $300 \mathrm{~K}$ predicted by our model is within the range of the transformation pressures reported by different authors and is very close to the transition pressure of 3.9 GPa reported by Olinger et al [43].

As the temperature increases, the thermodynamic driving force needed for the formation of omega phase decreases (Table-1). A negative value of the driving force indicates large driving force, whereas a large positive value indicates very small driving force. Hence a higher pressure is required in order to start the phase transformation at higher temperatures. Thus transformation start pressures increase with increasing temperature as indicated by the $\alpha-\omega$ equilibrium phase boundaries (Fig. 5). Although the overall trend is in agreement with the equilibrium phase boundaries, we observe that the transformation start pressures slightly decrease in the 473 - $673 \mathrm{~K}$ regime. These results are in accordance with experimental measurements of Zhang et al. and Olinger et al. $[34,43]$. We believe that this is a kinetic effect, in agreement with Ref. [38].

At $473 \mathrm{~K}$, we observe $31 \%$ volume fraction of $\omega$ at $5.75 \mathrm{GPa}$, whereas Wenk et al.'s DAC experiments report $77 \%$ volume fraction of $\omega$ at $5.2 \mathrm{GPa}$ [3]. Our simulations show that at $473 \mathrm{~K}, 100 \%$ volume fraction of $\omega$ is obtained at 8 GPa, whereas Wenk et al.'s DAC experiments report the pressure to be 12.2 GPa (Fig. 5).

Despite the assumptions made in the present model, e.g. isotropic elasticity and plasticity, the model predictions are consistent with experimental measurements.

\subsection{Prediction of structure-property relations}

The volume fraction-time plot shown in Fig. 6a shows the changes in volume fraction of $\omega$ during the forward transformation $(\alpha-\omega)$ and reverse transformations $(\omega-\alpha)$ at $300 \mathrm{~K}$ and $4 \mathrm{GPa}$. The forward transformation starts after some soaking time, whereas the reverse transformation starts instantaneously when the applied hydrostatic pressure is released. The change in volume fraction of $\omega$ during reverse transformation is in agreement with that observed during isothermal annealing experiments performed on shocked $\mathrm{Zr}$ samples [36]. The time-independent nature of the curves indicates that both transformations are of athermal type. Although some experimental studies indicate that the hydrostatic pressure-induced $\alpha-\omega$ phase transformation in $\mathrm{Zr}$ is isothermal in nature, there are some reports that suggest that the transformation is athermal or a combination of isothermal-athermal process [5, 38].

As shown in Fig. 6b and c, von Mises equivalent stress increases initially and then slightly decreases indicating local softening of the crystal during the $\alpha-\omega$ transformation. Thereafter, stress increases with increasing strain due to the growth of $\omega$, which induces stresses and strains in the material. During the early stages of the reverse transformation $(\omega-\alpha)$, the stress increases with decreasing strain. This can be attributed to the resistance (energy barrier) offered by the hard and brittle $\omega$ laths to the formation of soft and ductile $\alpha$. However during 


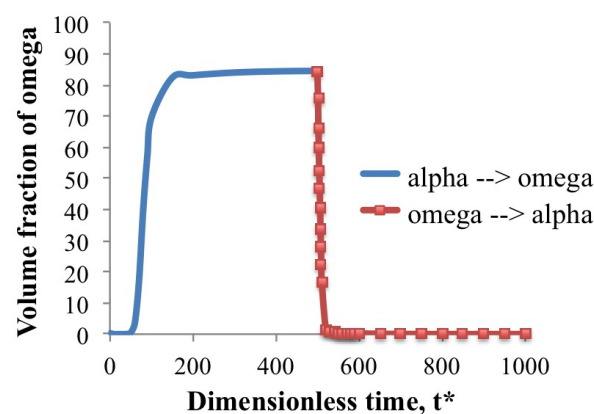

(a)

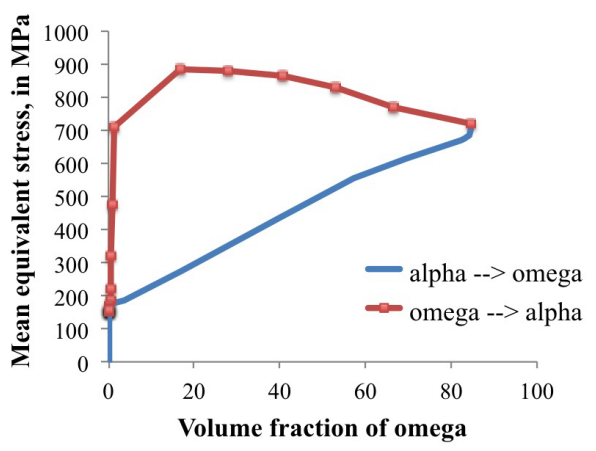

(c)

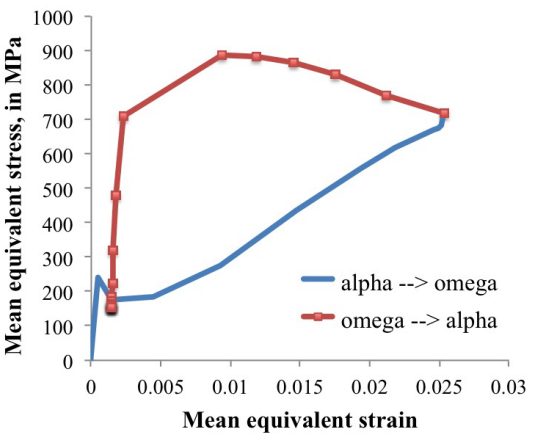

(b)

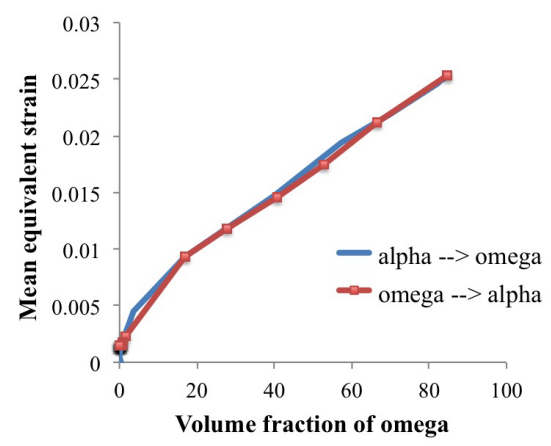

(d)

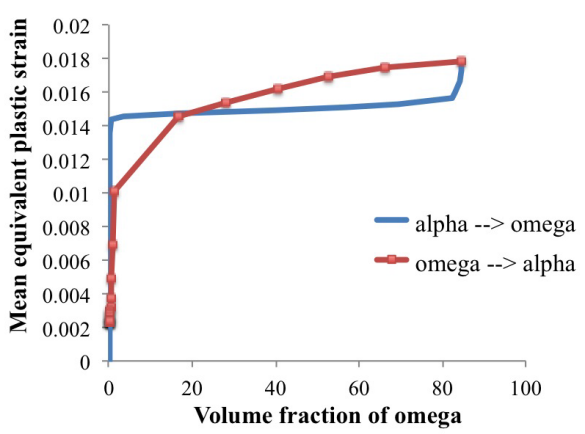

(e)

Figure 6: Plots of (a) $\omega$ volume fraction with time $t^{*}$ (b) mean von Mises equivalent stress - strain (c) $\omega$ volume fraction with mean von Mises equivalent stress (d) $\omega$ volume fraction with mean von Mises equivalent strain (e) $\omega$ volume fraction with mean von Mises equivalent plastic strain. Both $\alpha-\omega$ and $\omega-\alpha$ phase transformations show athermal behavior. Equivalent stresses and plastic strains show internal hysteresis. Total strain does not show any internal hysteresis because of small plastic strains induced into the material.

the later stages of the reverse transformation, the resistance decreases due to the decrease in volume fraction of $\omega$. Hence, the system overcomes the energy barriers and $\omega$ transforms easily to $\alpha$, thereby causing stress relaxation. Some 
stresses are retained in the material although $\omega$ completely reverts to $\alpha$.

As shown in Fig. 6d and e, the von Mises equivalent strain and plastic strain increase with increasing volume fraction of $\omega$ during the $\alpha-\omega$ transformation. The growth of $\omega$ in the $\alpha$ matrix induces strains and plastic strains and hence the strains increase during $\alpha-\omega$ transformation. However, the plastic strain does not increase significantly with increasing volume fraction, except the initial sudden increase due to the instantaneous application of hydrostatic pressure. During the $\omega-\alpha$ transformation, a decrease in volume fraction of $\omega$ relaxes the strains and plastic strains in the material and hence they decrease.

Fig. $6 \mathrm{~b}$ and $\mathrm{c}$ shows that the material behavior is not identical during the application of pressure and release of pressure, indicating internal hysteresis. Fig. 6d shows that total strain does not show any internal hysteresis, whereas Fig. 6e shows that plastic strain exhibits little internal hysteresis. As the plastic strains induced into the material are very low and since plastic strains do not increase drastically with increasing volume fraction of $\omega$ during the $\alpha-\omega$ transformation (Fig. 6e), plastic strain does not significantly affect the total strain in the material. Hence the total strain does not show any internal hysteresis.

\section{Conclusions}

Our model predicts that $\alpha-\omega$ phase transformation in $\mathrm{Zr}$ takes place at $300 \mathrm{~K}$ under hydrostatic pressure of $4 \mathrm{GPa}$. On application of hydrostatic pressure, three different crystallographic $\omega$ variants form in the shape of lath-like units. Autocatalysis, i.e. minimization of stresses by nucleation of several omega variants, and plastic deformation relax the stresses induced by the growing brittle $\omega$ and facilitate the phase transformation.

On sudden release of hydrostatic pressure, $\omega$ becomes unstable and reverts to $\alpha$ in a martensitic manner. Our simulations predict that both $\alpha-\omega$ and $\omega-\alpha$ phase transformations occur at $4 \mathrm{GPa}$, which is in good agreement with experimental results $[38,49]$. We observe that $\omega$ completely reverts to $\alpha$, although experiments report that only a fraction of $\omega$ reverts to $\alpha$. The differences are due to the defect-free single crystal considered in the present study. Reversion initiates at the lath boundaries since these are the areas where a low nucleation energy is sufficient to overcome the transformation barriers. Only a negligible amount of plastic strains are retained in the material after the reversion of $\omega$ to $\alpha$ indicating a perfect memory, in agreement with experimental data [3].

For each given temperature, the transformation start pressures are predicted and a phase diagram is plotted. The predicted transformation start pressure of 4 $\mathrm{GPa}$ at $300 \mathrm{~K}$ is very close to the $\omega$-start pressure of $3.9 \mathrm{GPa}$ at $297 \mathrm{~K}$, measured by Olinger et al. [43]. The transformation start pressures increase with increasing temperature. However, the transformation start pressures slightly decrease in the $473-673 \mathrm{~K}$ regime, due to a kinetic effect. The model predictions are in agreement with experimental results $[34,38,43]$.

The $\omega$ volume fraction - time plots indicate that the transformation proceeds in an athermal manner during both forward and reverse transformations. The 
growth of $\omega$ during forward transformation induces stresses, strains and plastic strains in the material. Small stresses, strains and plastic strains are retained in the material even after $\omega$ completely reverts to $\alpha$. The material behavior is not identical during the forward and reverse phase transformations, indicating internal hysteresis. However, the total strains do not show any internal hysteresis.

Despite the assumptions made in the present model, e.g. isotropic elasticity and plasticity, the model predictions are in reasonable agreement with experimental results. In order to compare the simulated microstructures with real microstructures, there is a need for in-depth electron back scattered diffraction (EBSD) studies of microstructures obtained in $\mathrm{Zr}$ under hydrostatic pressure.

\section{Acknowledgements}

This work was supported by the U.S. Department of Energy. This research used resources provided by the Los Alamos National Laboratory Institutional Computing Program, which is supported by the U.S. Department of Energy under Contract No. DE-AC52-06NA25396.

\section{References}

[1] R. Tewari, D. Srivastava, G.K. Dey, J.K. Chakravarty, S. Banerjee, Microstructural evolution in zirconium based alloys, J. Nucl. Mat. 383 (2008) 153-171.

[2] S. Banerjee, R. Tewari, G.K. Dey GK, Omega phase transformation - morphologies and mechanisms, Int. J. Mat. Res. 97 (2006) 963-977.

[3] H.R. Wenk, P. Kaercher, W. Kanitpanyacharoen, E. Zepeda-Alarcon, Y. Wang, Orientation relations during the alpha - omega phase transition of zirconium: in situ texture observations at high pressure and temperature, Phys. Rev. Lett. 111 (2013) 195701.

[4] D. Ping, Review on omega phase in body-centered cubic metals and alloys, Acta Metall. Sin. 27 (2014) 1-11.

[5] S.K. Sikka, Y.K. Vohra, R. Chidambaram, Omega phase in materials, Prog. Mat. Sci. 27 (1982) 245-310.

[6] D. Errandonea, Y. Meng, M. Somayazulu, D. Hausermann, Pressureinduced alpha - omega transition in titanium metal: a systematic study of the effects of uniaxial stress, Physica B 355 (2005) 116-125.

[7] M.X. Huang, O. Bouaziz, D. Barbier, S. Allain, Modelling the effect of carbon on deformation behavior of twinning induced plasticity steels, J. Mater. Sci. 46 (2011) 7410-7414. 
[8] H. Hallberg, P. Håkansson, M. Ristinmaa, A constitutive model for the formation of martensite in austenitic steels under large strain plasticity, Int. J. Plast. 23 (2007) 1213-1239.

[9] A.M. Beese, D. Mohr, Anisotropic plasticity model coupled with Lode angle dependent strain-induced transformation kinetics law, J. Mech. Phys. Solids 60 (2012) 1922-1940.

[10] L.Q. Chen, Phase-field models for microstructure evolution, Annu. Rev. Mater. Res. 32 (2002) 113-140.

[11] S. Minamoto, S. Nomoto, A. Hamaya, T. Horiuchi, S. Miura, Microstructure simulation for solidification of magnesium-zinc-yttrium alloy by multiphase-field method coupled with CALPHAD database, ISIJ Int. 50 (2010) 1914-1919.

[12] A.G. Khachaturyan, Theory of Structural Transformations in Solids, Dover Publications, New York; 2008.

[13] S.R. Shenoy, T. Lookman, A. Saxena, A.R. Bishop, Martensitic textures: Multiscale consequences of elastic compatibility, Phys. Rev. B 60 (1999) $12537-12541$.

[14] H.K. Yeddu, A. Malik, J. Ågren, G. Amberg, A. Borgenstam, Threedimensional phase-field modeling of martensitic microstructure evolution in steels, Acta Mater. 60 (2012) 1538-1547.

[15] Y. Wang, A.G. Khachaturyan, Three-dimensional field model and computer modeling of martensitic transformations, Acta Mater. 45 (1997) 759773.

[16] H.K. Yeddu, A. Borgenstam, P. Hedström, J. Ågren, A phase-field study of the physical concepts of martensitic transformations in steels, Mater. Sci. Eng. A 538 (2012) 173-181.

[17] A. Artemev, Y. Jin, A.G. Khachaturyan, Three-dimensional phase field model of proper martensitic transformation, Acta Mater. 49 (2001) 11651177.

[18] X.H. Guo, S.Q. Shi, Q.M. Zhang, X.Q. Ma, An elastoplastic phase-field model for the evolution of hydride precipitation in zirconium. Part I: Smooth specimen, J. Nucl. Mat. 378 (2008) 110-119.

[19] A. Malik, H.K. Yeddu, G. Amberg, A. Borgenstam, J. Ågren, Three dimensional elasto-plastic phase field simulation of martensitic transformation in polycrystal. Mater. Sci. Eng. A 556 (2012) 221-232.

[20] R. Ahluwalia, T. Lookman, A. Saxena, R.C. Albers, Landau theory for shape memory polycrystals, Acta Mater. 52 (2004) 209-218. 
[21] H.K. Yeddu, A. Borgenstam, J. Ågren, Stress-assisted martensitic transformations in steels: A 3-D phase-field study, Acta Mater. 61 (2013) 25952606.

[22] R. Ahluwalia, T. Lookman, A. Saxena, Dynamic strain loading of cubic to tetragonal martensites, Acta Mater. 54 (2006) 2109-2120.

[23] H.K. Yeddu, V.I. Razumovskiy, A. Borgenstam, P.A. Korzhavyi, A.V. Ruban, J. Ågren, Multi-length scale modeling of martensitic transformations in stainless steels, Acta Mater. 60 (2012) 6508-6517.

[24] A. Yamanaka, T. Takaki, Y. Tomita, Elastoplastic phase-field simulation of self- and plastic accommodations in Cubic - tetragonal martensitic transformation, Mater. Sci. Eng. A 491 (2008) 378-384.

[25] H.K. Yeddu, T. Lookman, A. Saxena, Strain-induced martensitic transformation in stainless steels: A three-dimensional phase-field study, Acta Mater. 61 (2013) 6972-6982.

[26] H.K. Yeddu, T. Lookman, Phase-field modeling of the beta to omega phase transformation in Zr-Nb alloys, Mater. Sci. Eng. A 634 (2015) 46-54.

[27] T.W. Heo, L.Q. Chen, Phase-field modeling of displacive phase transformations in elastically anisotropic and inhomogeneous polycrystals, Acta Mater 76 (2014) 68-81.

[28] H.K. Yeddu, T. Lookman, A. Saxena, Reverse phase transformation of martensite to austenite in stainless steels - A phase-field study, J. Mater. Sci. 49 (2014) 3642-3651.

[29] M. Mamivand, M.A. Zaeem, H.E. Kadiri, Shape memory effect and pseudoelasticity behavior in tetragonal zirconia polycrystals: A phase field study, Int. J. Plast. 60 (2014) 71-86.

[30] H.K. Yeddu, T. Lookman, A. Saxena, The simultaneous occurrence of martensitic transformation and reversion of martensite, Mater. Sci. Eng. A 594 (2014) 48-51.

[31] J. Kundin, D. Raabe, H. Emmerich, A phase-field model for incoherent martensitic transformations including plastic accommodation processes in the austenite, J. Mech. Phys. Solids 59 (2011) 2082-2102.

[32] H.K. Yeddu, A. Borgenstam, J. Ågren, Effect of martensite embryo potency on the martensitic transformations in steels - A 3D phase-field study, J. Alloys Compd. 577S (2013) S141-S146.

[33] X.H. Guo, S.Q. Shi, X.Q. Ma, Elastoplastic phase field model for microstructure evolution, Appl. Phys. Lett. 87 (2005) 221910. 
[34] J. Zhang, Y. Zhao, C. Pantea, J. Qian, L.L. Daemen, P.A. Rigg, R.S. Hixson, C.W. Greeff, G.T. Gray, Y. Yang, L. Wang, Y. Wang, T. Uchida, Experimental constraints on the phase diagram of elemental zirconium, J. Phys. Chem. Solids 66 (2005) 1213-1219.

[35] S.M. Allen, J.W. Cahn, A microscopic theory for antiphase boundary motion and its application to antiphase domain coarsening, Acta Metall. 27 (1979) 1085-1095.

[36] H. Zong, T. Lookman, X. Ding, C. Nisoli, D. Brown, S.R. Niezgoda, S. Jun, The kinetics of the alpha to omega phase transformation in $\mathrm{Zr}$, Ti: Analysis of data from shock-recovered samples and atomistic simulations, Acta Mater. 77 (2014) 191-199.

[37] E.A. De Souza Neto, D. Peric, D.R.J. Owen, Computational methods for plasticity-Theory and applications, John Wiley and Sons Ltd., West Sussex (UK), 2008.

[38] Y.K. Vohra, Kinetics of phase transformations in Ti, Zr and Hf under static and dynamic pressures, J. Nucl. Mat. 75 (1978) 288-293.

[39] B. Tang, Y.W. Cui, H. Chang, H.Kou, J.Li, L.Zhou, A phase-field approach to athermal beta-omega transformation, Comp. Mater. Sci. 53(2012) 187193.

[40] G. Amberg, R. Tönhardt, C. Winkler, Finite element simulations using symbolic computing, Math. and Comp. in Simul. 49 (1999) 257-274.

[41] M. Do-Quang, W. Villanueva, I. Singer-Loginova, G. Amberg, Parallel adaptive computation of some time-dependent materials-related microstructural problems, Bull. Pol. Acad. Sci-Tech. Sci. 55 (2007) 229-237.

[42] www.thermocalc.com

[43] B. Olinger, J.C. Jamieson, Zirconium: phases and compressibility to 120 kilobars, High Temp. - High Pressures 5 (1973) 123-131.

[44] W. Liu, B. Li, L. Wang, J. Zhang, Y. Zhao, Simultaneous ultrasonic and synchrotron $\mathrm{x}$-ray studies on pressure induced alpha-omega phase transition in zirconium, J. Appl. Phys. 104 (2008) 076102.

[45] Y. Zhao, J. Zhang, Enhancement of yield strength in zirconium metal through high-pressure induced structural phase transition, Appl. Phys. Lett. 91 (2007) 201907.

[46] S.R. Chen, G.T. Gray III, Influence of twinning on the constitutive responses of Zr: experiments and modeling, J. Phys. IV France 7 (1997) C3-741-746. 
[47] A. Rabinkin, M. Talianker, O. Botstein, Crystallography and a model of the alpha-omega phase transformation in zirconium, Acta Metall. 29 (1981) 691-698.

[48] S.G. Song, G.T. Gray III, Microscopic and crystallographic aspects of retained omega-phase in shock-loaded zirconium and its formation mechanism, Phil. Mag. A 71 (1995) 275-290.

[49] V.A. Zilbershtein, N.P. Chistotina, A.A. Zharov, N.S. Grishina, E.I. Estrin, Alpha-omega transformation in titanium and zirconium during shear deformation under pressure, Fiz. Met. Metal. 39 (1975) 445-447.

[50] W. Liu, B. Li, L. Wang, J. Zhang, Y. Zhao, Elasticity of omega phase zirconium, Phys. Rev. B 76 (2007) 144107. 


\section{Graphical Abstract}

Microstructure evolution during $\alpha-\omega$ phase transformation under hydrostatic pressure

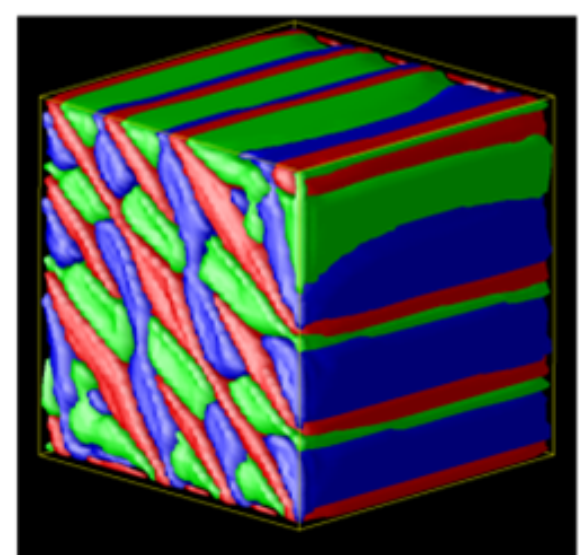

Microstructure evolution during $\omega-\alpha$ phase transformation on pressure release

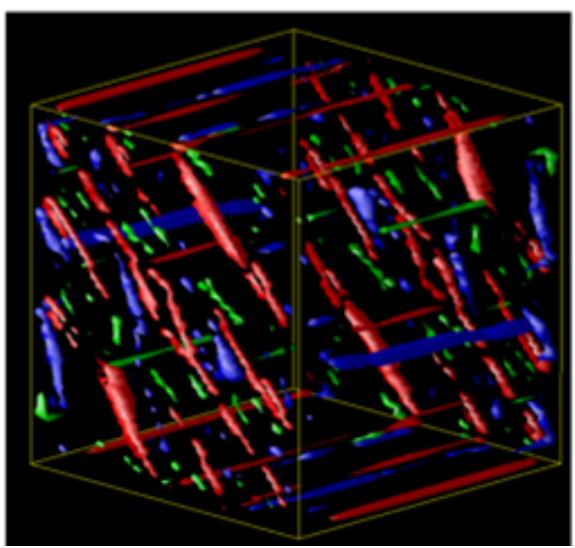

